\title{
Data quality: a survey of data quality dimensions
}

\begin{abstract}
Nowadays, activities and decisions making in an organization is based on data and information obtained from data analysis, which provides various services for constructing reliable and accurate process. As data are significant resources in all organizations the quality of data is critical for managers and operating processes to identify related performance issues. Moreover, high quality data can increase opportunity for achieving top services in an organization. However, identifying various aspects of data quality from definition, dimensions, types, strategies, techniques are essential to equip methods and processes for improving data. This paper focuses on systematic review of data quality dimensions in order to use at proposed framework which combining data mining and statistical techniques to measure dependencies among dimensions and illustrate how extracting knowledge can increase process quality.
\end{abstract}

Keyword: Data quality; Data quality dimensions; Types of data 